\title{
SOLAR WIND FROM CORONAL FUNNELS AND TRANSITION REGION Ly $\alpha$
}

\author{
RUTH ESSER \\ Harvard-Smithsonian Center for Astrophysics, 60 Garden Street, Cambridge, MA 02138; resser@cfa.harvard.edu \\ ØYSTEIN LIE-SVENDSEN ${ }^{2}$ \\ Norwegian Defence Research Establishment, P.O. Box 25, N-2027 Kjeller, Norway; oystein.lie-svendsen@ ffi.no \\ AND \\ Åse Marit Janse ${ }^{2}$ And Mari Anne Killie ${ }^{2}$ \\ Institute of Theoretical Astrophysics, University of Oslo, P.O. Box 1029, Blindern, N-0315 Oslo, Norway; a.m.janse@astro.uio.no, m.a.killie@astro.ui.no \\ Received 2005 June 1; accepted 2005 July 5; published 2005 July 26
}

\begin{abstract}
Using a newly developed gyrotropic solar wind model that extends continuously from the mid-chromosphere to $1 \mathrm{AU}$ and that accounts for radiative losses in the transition region, we have studied the difference between the fast solar wind emanating from a funnel geometry and a "traditional" rapidly expanding wind. The main aim is to determine whether or not observations of the Ly $\alpha$ intensity in the low transition region can be reconciled with solar wind models. In a rapidly expanding geometry, we are not able to produce a Ly $\alpha$ intensity much higher than $1 / 10$ of the observed values without creating a large pressure in the transition region and, as a result, a mass flux much higher than observed. In a funnel, on the other hand, we can easily obtain the observed Ly $\alpha$ intensity, while still having a wind solution in agreement with observations. The main reason for this is that the fast flow in the funnel causes hydrogen to be very far from ionization equilibrium, with the Ly $\alpha$ intensity coming from temperatures of about $5 \times 10^{4} \mathrm{~K}$. At these elevated temperatures, the radiative loss is much more efficient. The results of this Letter support the idea that the solar wind originates from small coronal funnels.
\end{abstract}

Subject headings: solar wind — Sun: corona — Sun: transition region

\section{INTRODUCTION}

The high-speed solar wind originates in coronal holes. Recent observations of large blueshifts above chromospheric network lanes (e.g., Hassler et al. 1999; Peter \& Judge 1999; Wilhelm et al. 2000; Xia et al. 2003; Tu et al. 2005), interpreted as large outflow speeds, suggest that the fast wind originates in coronal funnels (e.g., Peter 2001), very much in agreement with the geometry suggested by Dowdy et al. (1986) and modeled by Marsch \& Tu (1997) and Hackenberg et al. (2000). These outflow speeds are of order $5-7 \mathrm{~km} \mathrm{~s}^{-1}$ for the lines originating from plasma at about $8 \times 10^{4}-2 \times 10^{5} \mathrm{~K}$, and $5-$ $20 \mathrm{~km} \mathrm{~s}^{-1}$ above.

At such high outflow speeds, hydrogen and other elements are significantly out of ionization equilibrium. The Ly $\alpha$ spectral line radiation, formed between $8 \times 10^{3}$ and $2 \times 10^{4} \mathrm{~K}$ in equilibrium and the strongest radiation by far, is shifted to higher temperatures where the electron excitation rate that causes the Ly $\alpha$ emission is much enhanced. In this Letter, we investigate the increase of the Ly $\alpha$ intensity that can be expected from a fast funnel flow as compared to a slower flow in a geometry that expands only a factor of 5 faster than radial.

\section{MODEL}

We use a newly developed set of gyrotropic transport equations (Killie et al. 2004; Janse et al. 2005) that yield a better description of the collision-dominated region of the plasma flow, in agreement with classical transport theory, while retaining the form for the collisionless regime of Demars \& Schunk (1979). For a hydrogen-proton-electron plasma, in which thermal diffusion does not play a role, the main improvement lies in the description of heat conduction. For strictly

\footnotetext{
${ }^{1}$ On leave from the University of Troms $\varnothing$, N-9037 Tromsø, Norway.

${ }^{2}$ Visiting Physicist, Harvard-Smithsonian Center for Astrophysics.
}

radial flow, this set reduces to five coupled equations describing the transport of mass, momentum, energy (separate temperatures $\|$ and $\perp$ to the magnetic field), and heat flux. The model extends continuously from the mid-chromosphere to $1 \mathrm{AU}$, includes radiative losses (see below), and allows the transition region to adjust itself to achieve energy balance using an adaptive grid. In this type of model, also called a radiative energy balance model (Withbroe 1988), only the heating parameters and the geometry can be chosen. All the other parameters, including the location of the transition region, come out of the model. Details of the numerical scheme are given by LieSvendsen et al. (2001).

All suggested coronal heating mechanisms rely on ad hoc assumptions. We investigate whether or not the observed Ly $\alpha$ radiation can be made consistent with the observed properties of the corona and wind. A close fit to observed quantities is, therefore, more important than the particular heating mechanisms. To achieve this fit, we have chosen exponential damping of a prescribed energy flux for each species. In the extended corona and wind, we assume proton heating/acceleration by a turbulent cascade of Alfvén waves (Hollweg 1986).

For the flow tube, which is $1 \mathrm{~m}^{2}$ at the lower boundary, we used a Kopp \& Holzer (1976) expression. The rapid expansion, called hole, opens 5 times more than radial. For the funnel, we take parameters inherent in models explaining the first ionization potential (FIP) effect that are in the range of 3-96 for the initial expansion and 7 for the expansion farther up (see Peter \& Marsch 1998). We have used 62 and 7, respectively (Fig. 1, lowest panel). This expansion is larger than the extrapolated values given by Tu et al. (2005); however, we want to demonstrate a principle for the $\operatorname{Ly} \alpha$ intensity. Also, the true expansion of the funnel is not yet known.

To calculate the radiative losses from hydrogen, ideally one should solve the equation of radiative transfer. However, combining the radiative transport with a detailed description of 

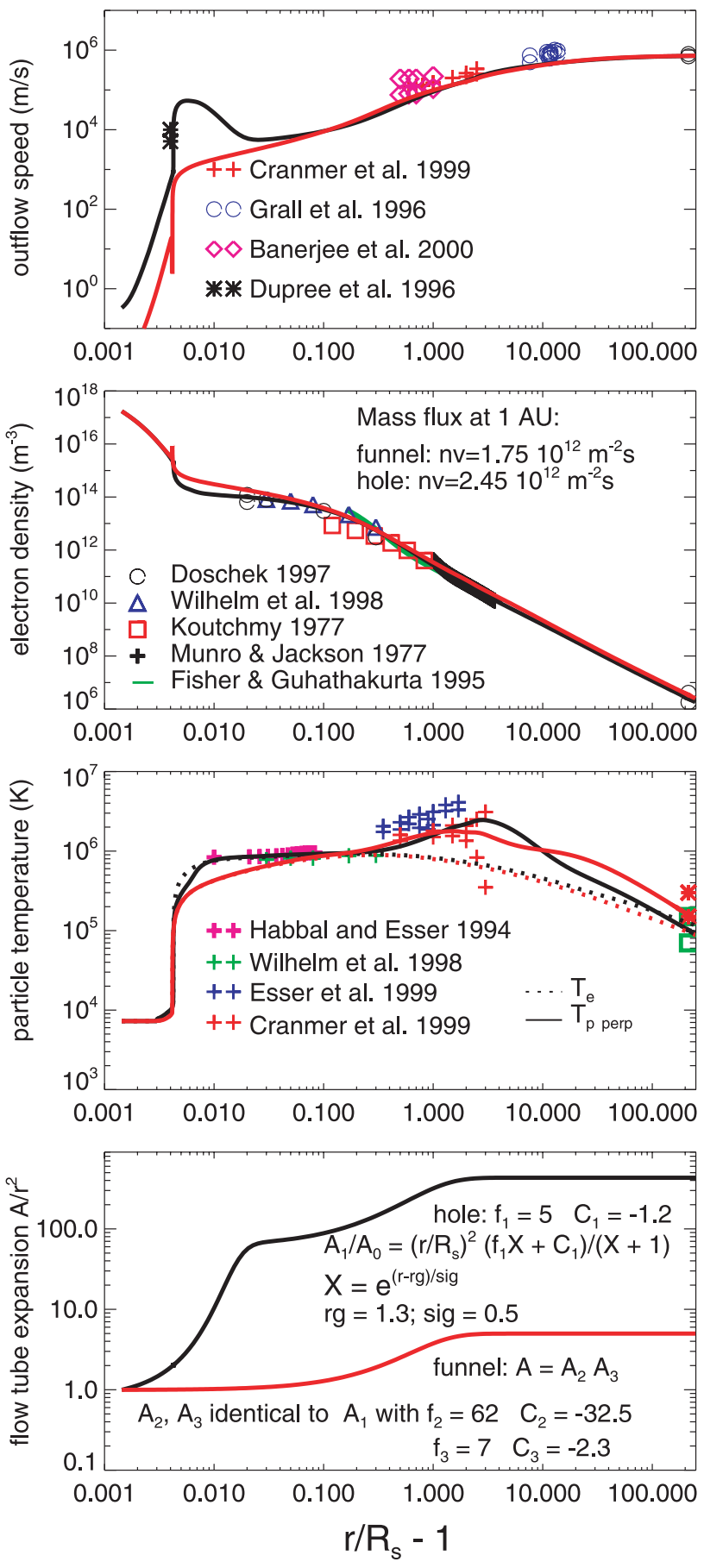

FIG. 1.-Solutions for the funnel (black lines) and hole (red lines). In situ observations (symbols at $215 R_{S}$ ) are from McComas et al. (2000).

plasma dynamics and thermodynamics in a full solar wind model is presently not possible. We, therefore, assume that the Ly $\alpha$ line is optically thin. However, we do use the hydrogen population densities calculated in the model. Comparison to the radiation calculated using the radiative transport equation (Kuin \& Poland 1991) indicates that the errors due to the assumption of effectively thin losses in $\operatorname{Ly} \alpha$ are relatively small. Radiative losses due to thermal bremsstrahlung and $\mathrm{C}, \mathrm{O}, \mathrm{Ne}$, and $\mathrm{Fe}$ are also included, with atomic data and collisional excitation rates from Arnaud \& Rothenflug (1985), Shull \& van Steenberg (1982), and Judge \& Meisner (1994).

\section{RESULTS}

The model results are shown in Figure 1 together with a large number of observations. In the funnel, the outflow speed is much higher in the region below about $1.02 R_{S}\left(R_{S}\right.$ is the solar radius) due to the much larger expansion in that geometry and mass flux conservation. The decrease of the flow speed between about 1.005 and $1.02 R_{S}$ leads to a flatter density profile in that region in the funnel than in the hole, which agrees well with observations by Doschek et al. (1997) and Wilhelm et al. (1998).

In the inner corona, the temperature is significantly higher in the funnel than in the hole and fits well with the electron temperature derived from SUMER (Wilhelm et al. 1998) and Skylab (Habbal \& Esser 1994). To achieve this fit, about $7 \%$ of the energy was put into the electrons in this region. In the hole, $15 \%$ of the energy was deposited here, and still the temperature is much lower. We are not able to increase the hole temperature without increasing the pressure, which in turn leads to an unacceptably large mass flux. Hence, the coronal observations of electron density and temperature seem to be more easily fit using a funnel.

The reason for the difference in electron temperature is the different downward heat conduction in the two geometries. Neglecting the solar wind energy loss, which is quite similar in both cases, the temperature of the inner corona is set by the balance between heating and downward heat conduction. For the argument here, assume that the funnel and hole flow tubes have the same area in the inner corona, that the funnel then shrinks to a much smaller area in the transition region (while the hole flow tube area is essentially constant between the chromosphere and the inner corona), and that the same energy flux is to be conducted downward in both geometries. In the funnel, the heat flux density must then increase as the area shrinks (energy flux conservation); thus, the temperature gradient must become steeper at each height. Starting at a given temperature level in the transition region (sufficiently high that radiation is unimportant), the funnel temperature must then increase more steeply, and the two temperature curves will never cross above this level (because that would require the funnel temperature gradient to be less steep at the crossing point). Hence, the funnel must have a higher corona temperature than the hole. Alternatively, for a given corona temperature, less heat will be conducted into the funnel than into a constant area flow tube (e.g., for a dipole magnetic field with $A_{1} / A_{0} \gg 1$, the downward heat flux is proportional to $\left(A_{0} / A_{1}\right)^{2 / 3}$, where $A_{0}$ and $A_{1}$ are the footpoint and coronal areas, respectively). This is the reason why we are able to match the high electron temperatures in the funnel. Despite the higher temperature, the downward heat flux density is only about half of the hole value at these upper heights.

Figure 2 shows the energy fluxes. Since the funnel has a much larger area at $1 \mathrm{AU}$, the energy fluxes in the two geometries cannot be compared directly. The innermost energy input is required by the observed electron temperatures (see above) and the outermost Alfvén wave heating/acceleration by the maximum observed proton temperature, which is of order $3 \times 10^{6} \mathrm{~K}$ and not enough to produce a high-speed wind. Thus, some additional energy has to be applied in the outer regions. In the hole, most of the heat conducted downward from the corona (about $60 \mathrm{~W}$ ) is radiated away, and a negligible fraction of the energy goes into heating the upstreaming plasma (enthalpy flux), hence the expression radiative energy balance model. In the funnel, on the other hand, most of the energy 


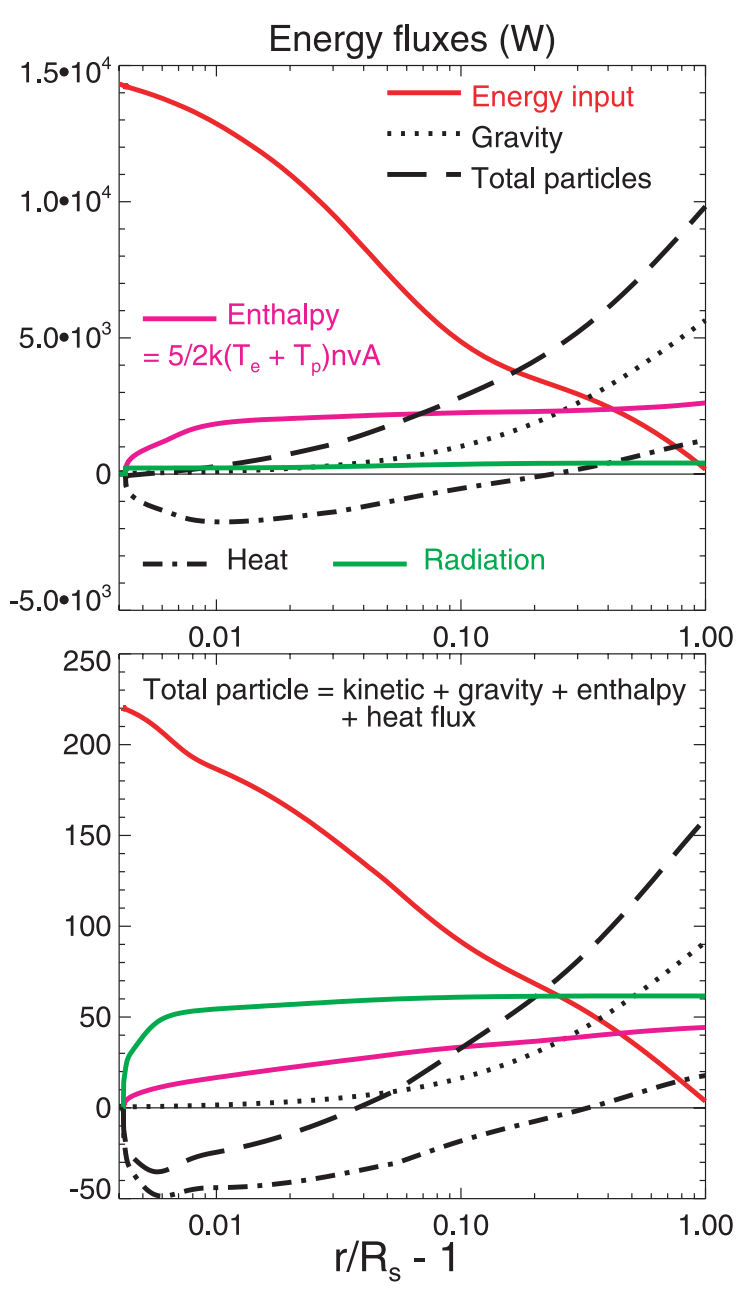

FIG. 2.-Energy flux densities multiplied by the flow tube areas and cumulative radiation losses integrated over the flux tube volume for the funnel (upper panel) and hole (lower panel). The energy input is normalized to the value at $r / R_{S}=2$.

coming down from the corona (about $2000 \mathrm{~W}$ ) goes into enthalpy flux, and a much smaller fraction goes into radiation.

Figure 3 shows the Ly $\alpha$ loss rate and the electron temperature. The $\mathrm{Ly} \alpha$ comes from a rather narrow part of the transition region. Due to the high outflow speed at temperatures below $10^{5} \mathrm{~K}$, hydrogen is much more out of ionization equilibrium in the funnel. The peak of the Ly $\alpha$ loss rate is shifted from about $2.2 \times 10^{4} \mathrm{~K}$ in the hole to $4.8 \times 10^{4} \mathrm{~K}$ in the funnel. The excitation rate from the ground state to the first excited level of hydrogen can be written $L_{\alpha}=C \exp \left[-E_{\alpha} /\left(k T_{e}\right)\right]$, where $T_{e}$ is the electron temperature, $k$ is Boltzmann's constant, $E_{\alpha} \simeq 10.2 \mathrm{eV}$, and $C$ is only weakly dependent on temperature. At temperatures below $E_{\alpha} / k \sim 10^{5} \mathrm{~K}, L_{\alpha}$ increases rapidly with temperature, approximately a factor of 15 between the two loss rate maxima in Figure 3. Despite that the product of the electron and neutral hydrogen densities at these maxima are comparable in the two models (about a factor of 1.5 higher in the hole geometry), the maximum $\operatorname{Ly} \alpha$ radiation loss rate is therefore approximately a factor of 10 higher in the funnel than in the hole geometry.

To compare with observations, we have integrated the radiation losses along a vertical cylinder (Fig. 4). Here we can see the increase of the Ly $\alpha$ radiation in the funnel, which has a value of about $100 \mathrm{~W} \mathrm{~m}^{-2}$, close to the value estimated by
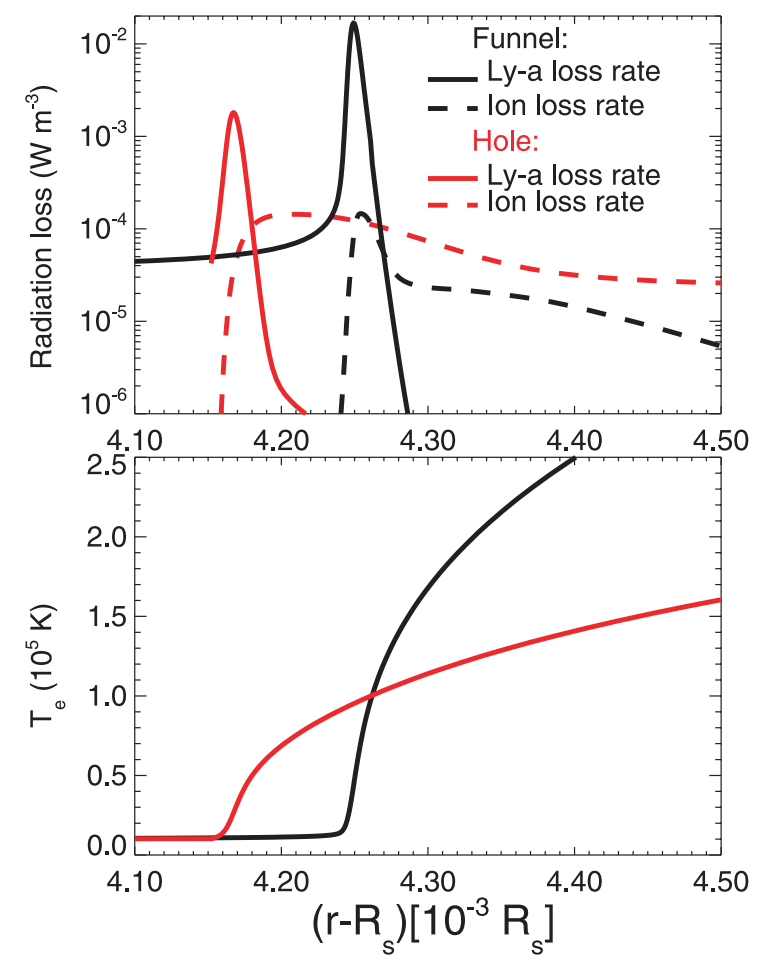

FIG. 3.-Radiation loss rates per unit volume due to Ly $\alpha$ and ions/bremsstrahlung (upper panel) and electron temperature (lower panel).

Dowdy et al. (1986) for radiation from a funnel. Also, the enthalpy flux is much increased in the funnel. To achieve a similar radiation loss in the hole, the transition region pressure would have to be increased to values about 3 times larger than observed, which would lead to a mass flux much higher than observed.

The total radiation, which includes the radiation from heavy ions and bremsstrahlung, is only roughly estimated here. Since we do not include the ions in the present model, we cannot calculate this part of the radiation with the actual ionization

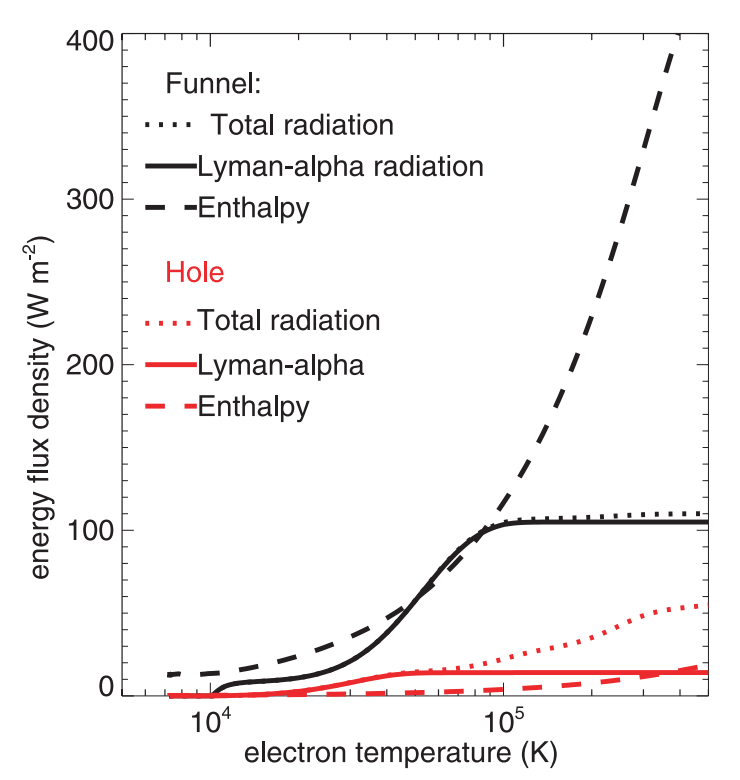

FIG. 4.-Cumulative radiation losses integrated along a vertical cylinder and enthalpy flux density for the funnel (black lines) and hole (red lines). 
state. However, it can be seen that the ion radiation comes from a much more extended part of the transition region than the Ly $\alpha$. Since the transition region in the funnel is much thinner, the flux from the heavy ions is less in the funnel than in the hole. Thus, the total radiation is even more dominated by Ly $\alpha$ in the funnel. However, this could be an artifact of assuming ionization equilibrium for the ions.

\section{SUMMARY}

Our results demonstrate that the $\operatorname{Ly} \alpha$ loss per unit area in a funnel is much higher than in a rapidly (or radially) expanding wind and can easily be reconciled with observations not only of the $\operatorname{Ly} \alpha$ intensity but also of the electron temperature in the inner corona. There are three reasons why a funnel solar wind can accommodate both a higher $\operatorname{Ly} \alpha$ radiative loss and a higher electron temperature. First, for a given temperature, the downward heat flux density in the corona will be smaller in a funnel than in a flow tube that expands less. Hence, we can increase the electron temperature and bring it into agreement with observations without increasing the mass flux. Second, although the heat flux density increases strongly farther down in the funnel (because the flow tube area diminishes), most of this energy is not converted into radiation but is used to heat the upwelling plasma (increase the enthalpy flux), and because the particle flux density must be large in the funnel to sustain the observed mass flux, a large energy flux density is needed. Third, because the flow in the funnel is so fast that hydrogen is way out of ionization equilibrium, the $\operatorname{Ly} \alpha$ loss takes place at a higher temperature where the rate for electron excitation causing the Ly $\alpha$ emission has increased by roughly a factor of 15 compared to the hole. The much improved efficiency of electrons to cool the plasma at this higher temperature implies that a lower electron density is needed to achieve the same degree of cooling. For this reason, the Ly $\alpha$ loss is 10 times higher in the funnel, despite that the pressure is quite similar in the two geometries and that the transition region is much thinner in the funnel.

In short, by bringing hydrogen so far out of ionization equilibrium, the funnel is able to produce a high Ly $\alpha$ intensity, in approximate agreement with observations, at a transition region (and corona) pressure that is sufficiently low to still agree with observed coronal hole pressure and in situ mass flux. It seems that the $\operatorname{Ly} \alpha$ and near-Sun electron temperature observations lend support to the idea that the fast solar wind originates in small funnels that are the building blocks of coronal holes and that merge to form the unipolar coronal holes.

This work was supported by the Research Council of Norway under grants 146467/420 and by NASA grant NAG5-10996.

\section{REFERENCES}

Arnaud, M., \& Rothenflug, R. 1985, A\&AS, 60, 425

Banerjee, D., Teriaca, L., Doyle, J. G., \& Lemaire, P. 2000, Sol. Phys., 194, 43

Cranmer, S. R., et al. 1999, ApJ, 511, 481

Demars, H. G., \& Schunk, R. W. 1979, J. Phys. D. Appl. Phys., 12, 1051

Doschek, G. A., Warren, H. P., Laming, J. M., Mariska, J. T., Wilhelm, K., Lemaire, P., Schuehle, U., \& Moran, T. G. 1997, ApJ, 482, L109

Dowdy, J. F., Jr., Rabin, D., \& Moore, R. L. 1986, Sol. Phys., 105, 35

Dupree, A. K., Penn, M. J., \& Jones, H. P. 1996, ApJ, 467, L121

Esser, R., Fineschi, S., Dobrzycka, D., Habbal, S. R., Edgar, R. J., Raymond,

J. C., Kohl, J. L., \& Guhathakurta, M. 1999, ApJ, 510, L63

Fisher, R. R., \& Guhathakurta, M. 1995, ApJ, 447, L139

Grall, R. R., Coles, W. A., Klinglesmith, M. T., Breen, A. R., Williams, P. J. S., Markkanen, J., \& Esser, R. 1996, Nature, 379, 429

Habbal, S. R., \& Esser, R. 1994, ApJ, 421, L59

Hackenberg, P., Marsch, E., \& Mann, G. 2000, A\&A, 360, 1139

Hassler, D. M., Dammasch, I. E., Lemaire, P., Brekke, P., Curdt, W., Mason, H. E., Vial, J.-C., \& Wilhelm, K. 1999, Science, 283, 810

Hollweg, J. V. 1986, J. Geophys. Res., 91, 4111

Janse, А̊. M., Lie-Svendsen, Ø., \& Leer, E. 2005, J. Plasma Phys., in press

Judge, P. G., \& Meisner, R. W. 1994, in Proc. Third SOHO Workshop, Solar Dynamic Phenomena and Solar Wind Consequences, ed. J. J. Hunt (ESA SP-373; Paris: ESA), 67
Killie, M. A., Janse, Å. M., Lie-Svendsen, Ø., \& Leer, E. 2004, ApJ, 604, 842 Kopp, R. A., \& Holzer, T. E. 1976, Sol. Phys., 49, 43

Koutchmy, S. 1977, Sol. Phys., 51, 399

Kuin, N. P. M., \& Poland, A. I. 1991, ApJ, 370, 763

Lie-Svendsen, Ø., Leer, E., \& Hansteen, V. H. 2001, J. Geophys. Res., 106, 8217

Marsch, E., \& Tu, C.-Y. 1997, Sol. Phys., 176, 87

McComas, D. J., et al. 2000, J. Geophys. Res., 105, 10419

Munro, R. H., \& Jackson, B. V. 1977, ApJ, 213, 874

Peter, H. 2001, A\&A, 374, 1108

Peter, H., \& Judge, P. G. 1999, ApJ, 522, 1148

Peter, H., \& Marsch, E. 1998, A\&A, 333, 1069

Shull, J. M., \& van Steenberg, M. 1982, ApJS, 48, 95

Tu, C.-Y., Zhou, C., Marsch, E., Xia, L.-D., Zhao, L., Wang, J.-X., \& Wilhelm, K. 2005, Science, 308, 519

Wilhelm, K., Dammasch, I. E., Marsch, E., \& Hassler, D. M. 2000, A\&A, 353,749

Wilhelm, K., Marsch, E., Dwivedi, B. N., Hassler, D. M., Lemaire, P., Gabriel, A. H., \& Huber, M. C. E. 1998, ApJ, 500, 1023

Withbroe, G. L. 1988, ApJ, 325, 442

Xia, L. D., Marsch, E., \& Curdt, W. 2003, A\&A, 399, L5 